\title{
Comportamento alimentar e imagem corporal entre estudantes de educação física
}

\author{
Eating disorders and body image among physical education students \\ Maria Lúcia Magalhães Bosi', Ronir Raggio Luiz², Kátia Yumi Uchimura³, Fátima Palha de Oliveira
}

\section{RESUMO}

Objetivo: Caracterizar práticas alimentares e possíveis fatores de risco associados a transtornos do comportamento alimentar entre estudantes de Educação Física em uma universidade pública do município do Rio de Janeiro. Método: Estudo seccional, elegendo-se como população-alvo um segmento de risco para o surgimento de transtornos alimentares. Foram aplicados os questionários Bulimic Investigatory Test Edinburgh (BITE), Eating Attitudes Test (EAT-26), Body Shape Questionnaire (BSQ) e uma variável que considera os dois instrumentos associados. Resultados: Detectou-se resultado positivo em 6,9\% (IC95\%: 3,6-11,7\%) no EAT-26. No BITE, para sintomas elevados e gravidade intensa, foram encontra-

\section{Palavras-chave}

Transtornos alimentares, anorexia nervosa, bulimia nervosa, imagem corporal.

\section{Key-words}

Eating disorders, anorexia nervosa, bulimia nervosa, body image. das prevalências de 5\% (IC95\%: 2,4-9,5\%) e 2,5\% (IC95\%: 0,7-6,3\%), respectivamente. Constatou-se que $26,29 \%$ das estudantes apresentavam comportamento alimentar anormal. Conclusão: Os resultados deste estudo indicam que se deve atentar para comportamentos alimentares de risco nesse grupo, justificando-se um olhar diferenciado em relação a esses futuros educadores.

\section{ABSTRACT}

Objective: To characterize eating practices and possible risk factors associated with eating disorders among physical education students in a public university of the Municipality of Rio de Janeiro, Brazil. Methods: Sectional study, electing as target population a risk group for the emergence of eating disorders. The questionnaires Bulimic Investigatory Test Edinburgh (BITE), Eating Attitudes Test (EAT-26), Body Shape Questionnaire (BSQ) and a variety that considers related issues were applied. Results: A positive result was detected in 6.9\% of cases (IC95\%: 3.6-11.7\%) on EAT-26. On BITE, for elevated symptoms and severe cases, a prevalence of 5\% was found (IC95\%: 2.4-9.5\%) and 2.5\% (IC95\%: 0.7-6.3\%), respectively. It was evidenced that 26.29\% of students presented abnormal eating behavior. Conclusion: The results of this study demonstrate the need to pay attention to risk eating behaviors in this group, being justified to take special care with these future health care educators.
1 Departamento de Saúde Comunitária da Faculdade de Medicina da Universidade Federal do Ceará (UFC).

2 Instituto de Estudos em Saúde Coletiva (IESC/CCS) da Faculdade de Medicina da Universidade Federal do Rio de Janeiro (UFRJ). 3 Ciências Médicas da UFC.

4 Departamento de Biociências da Atividade Física da Escola de Educação Física/Desportos da UFRJ. 


\section{INTRODUÇÃO}

O aumento da prevalência e da incidência de transtornos do comportamento alimentar (TCA) - como anorexia nervosa (AN) e bulimia nervosa (BN) - na população ocidental tem sido alvo de muitas controvérsias, pois o aumento da capacidade de detecção e o registro dos casos, nos últimos anos, têm contribuído para elevar os dados estatísticos e, de maneira contraditória, para confundir o entendimento da real evolução desse fenômeno na sociedade ${ }^{1,2}$.

Existe consenso, no entanto, de que tais problemas atinjam, predominantemente, adolescentes e mulheres adultas jovens $s^{1,3,4}$, com proporção média na relação entre homens e mulheres de 1:105, podendo variar entre 1:6 e 1:20, conforme o critério diagnóstico utilizado e a modalidade de TCA avaliada ${ }^{6,7}$.

Estima-se que o risco de desenvolver TCA pode estar presente em 20\% das mulheres jovens, por apresentarem comportamentos subclínicos precursores ${ }^{8-10}$, embora nem todas cheguem a desenvolvê-los. Walsh, Wheat e Freund ${ }^{4}$ consideram diabéticas e mulheres atletas como dois grupos de alto risco para o desenvolvimento de TCA e, entre universitárias, Fiates e Salles ${ }^{11}$ encontraram comportamento de risco em mais de $20 \%$ da população pesquisada. De forma geral, a prevalência de AN varia entre $0,5 \%$ e 3,7\% e de BN de $1,1 \%$ a $4,2 \%{ }^{7}$, apresentando elevado percentual não só nos países desenvolvidos, onde subsistem as características econômicas e socioculturais para o seu desencadeamento, como também nos países em desenvolvimento ${ }^{12}$. Em meninas adolescentes, os números podem ser substancialmente maiores. Segundo Fairburn e Harrison', nesse grupo a prevalência de AN pode variar de $0 \%$ a $7 \%$.

A prevalência dos TCA é ainda mais elevada em pessoas que exercem determinadas atividades (modelos, bailarinas, atletas e profissionais da área da saúde), provavelmente mais vulneráveis aos TCA pelo fato de sofrerem, mais intensamente, pressões associadas a padrões estéticos, às quais se responde por meio da exibição de um corpo magro $4,7,11$.

Estudos, como o de Fiates e Salles ${ }^{11}$, sugerem que indivíduos que apresentam preocupação com seu peso e imagem corporal costumam fazer a opção por área de estudo correlata, em função, justamente, desse interesse pessoal anteriormente manifesto.

Em atletas, a prevalência de TCA ainda não é suficientemente conhecida, mas alguns estudos disponíveis demonstraram a freqüência aumentada em certas modalidades desportivas ${ }^{3}$, em virtude, sobretudo, da influência do baixo peso corporal na determinação de uma melhor performance e da ênfase no controle de peso a que são submetidas, como salto de esqui, ciclismo de estrada, alpinismo, ginástica artística ou rítmica e corridas de longa distância ${ }^{13}$.
O aumento da participação feminina na prática de diversas modalidades permite estimar a extensão desse problema, pois, nos últimos 20 anos, essa participação cresceu $600 \%$, superando a marca de 1,9 milhão de atletas ${ }^{14}$. Nesse grupo, é comum a ocorrência de uma síndrome que agrega três componentes inter-relacionados: transtorno alimentar, amenorréia e osteoporose. É a chamada tríade da mulher atleta (TMA), que não é exclusividade de atletas de elite ${ }^{15}$.

Diante do exposto, o objetivo deste estudo é investigar a prevalência de comportamentos alimentares anormais e a satisfação com a imagem corporal entre universitárias de um curso de educação física situado em uma universidade do município do Rio de Janeiro (região Sudeste do Brasil).

\section{MÉTODOS}

Realizou-se um estudo de delineamento transversal com uma amostra constituída por 191 alunas universitárias do primeiro ao último semestre do curso de educação física de uma universidade pública situada no município do Rio de Janeiro.

A investigação realizou-se por meio de questionários auto-aplicáveis em versões traduzidas para o português ${ }^{16}$. A adesão das informantes foi espontânea, após conhecimento dos objetivos do estudo e isenta de qualquer forma de remuneração, não implicando qualquer risco de dano físico ou moral. Os instrumentos auto-aplicáveis foram numerados progressivamente, portanto, sem identificação ou assinatura, a fim de garantir o anonimato das participantes. As aplicações se deram nas instalações da instituição, utilizando-se horários previamente escolhidos, respeitando-se a disponibilidade das alunas.

Para avaliar as atitudes e os comportamentos típicos de AN e o índice de gravidade de preocupações que caracterizam os TCA, adotou-se o Eating Attitudes Test (EAT-26) ${ }^{17}$. O EAT-26 é um instrumento de auto-relato, reconhecido internacionalmente, empregado para avaliar e identificar padrões alimentares anormais, sendo também útil no acompanhamento da evolução de casos clínicos. O instrumento consta de 26 itens, com seis opções de resposta: sempre, muito freqüente, freqüentemente, algumas vezes, raramente e nunca, realizando-se a análise fatorial destes itens em três aspectos:

\section{Fator 1}

Dieta - os primeiros 13 itens que refletem recusa patológica às comidas de alto teor calórico e preocupações com a forma física.

\section{Fator 2}

Bulimia nervosa - os seis itens seguintes refletem pensamentos sobre comida e atitudes bulímicas. 


\section{Fator 3}

Controle oral - os últimos sete itens refletem o autocontrole em relação à comida e reconhecem pressões sociais no ambiente para ganhar peso.

Caso o escore total encontrado seja maior que 21, o EAT-26 é considerado positivo (EAT-26+), o que confirma a presença de atitudes alimentares anormais e risco para o desenvolvimento de TCA. Os autores que desenvolveram o EAT-26 utilizaram um ponto de corte (cut-off) de 20 escores. No presente estudo, foi adotado o cut-off de 21 pontos, pois este apresenta sensibilidade e especificidade altamente satisfatórias ${ }^{18,19}$, tendo sido adotado por outros autores brasileiros ${ }^{20,21}$, o que permite comparar os resultados.

Para avaliar a presença de comportamentos bulímicos e a intensidade destes foi adotado o Bulimic Investigatory Test Edinburgh - BITE)22. Trata-se de questionário auto-aplicável, composto de 33 questões e duas subescalas, sendo uma voltada para sintomas e a outra para a gravidade destes. Essas escalas são descritas a seguir, segundo Cordás e Hpchgraf $^{23}$. A escala de sintomas oferece três grupos de escores:

- Alto (20 pontos ou mais): é considerado um escore elevado com presença de comportamento alimentar compulsivo e grande possibilidade de preencher critérios diagnósticos para BN pelo DSM-III-R.

- Médio (10 a 19 pontos): sugere padrão alimentar não usual e, em geral, não estão presentes todos os critérios para bulimia.

- Baixo (abaixo de 10 pontos): dentro dos limites de normalidade.

A escala de gravidade do BITE é constituída pelos itens 6,7 e 27 do instrumento, e mede a gravidade do comportamento compulsivo pela freqüência de atitudes, sendo analisada para os casos em que a pontuação na escala de sintomas é superior a 10, ramificando-se em três estágios:

- Alto (escore $\geq 10$ pontos): indicam alto grau de gravidade, podendo apontar a presença de vômito psicogênico ou abuso de laxante sem comportamento compulsivo, e devem ser seguidos de entrevista diagnóstica com especialistas.

- Moderado (entre 5 e 9 pontos): são considerados clinicamente significativos e devem ser seguidos de entrevista diagnóstica com especialistas.

- Baixo (até 5 pontos): resultado clinicamente não significativo.

Para avaliar a insatisfação com a imagem corporal, utilizou-se o Body Shape Questionnaire (BSQ) (validado por Cooper et $a 1 .{ }^{24}$ ), que mede o grau de preocupação com a forma do corpo e a auto-depreciação em virtude da aparência física e da sensação de estar gorda. De acordo com Cordás e Neves ${ }^{16}$, o questionário distingue dois aspectos específicos da imagem corporal: a exatidão da estimativa do tamanho corporal e os sentimentos em relação ao corpo (insatisfação ou desvalorização da forma física). A classificação dos resultados é feita pelo total de pontos obtidos e reflete os níveis de preocupação com a imagem corporal. Resultados no intervalo menor ou igual a 80 pontos indicam um padrão de normalidade, sendo considerado ausência de insatisfação com a imagem corporal. Resultados entre 81 e 110 pontos classificam-se leve insatisfação com a imagem corporal; entre 111 e 140 classificam-se moderada insatisfação com a imagem corporal; e acima de 140 pontos a classificação é de presença de grave insatisfação com a imagem corporal.

O EAT-26, o BITE e o BSQ foram utilizados, neste estudo, em suas versões traduzidas para o português ${ }^{16}$. Esclarecemos que esses instrumentos de auto-relato são usados na clínica médica para triagem e definição de quadros de comportamento alimentar anormal e de outras comorbidades psiquiátricas, e que os participantes do estudo não foram submetidos à entrevista para diagnóstico.

Este estudo foi aprovado pela Comissão de Ética do Núcleo de Estudos de Saúde Coletiva do Hospital Clementino Fraga Filho, unidade vinculada a uma universidade pública situada no município do Rio de Janeiro, tendo sido realizado mediante a assinatura de termo de consentimento por todos os participantes, no qual constavam os procedimentos que seriam adotados e obtida a autorização dos voluntários para a divulgação dos resultados encontrados em publicações científicas. $\mathrm{O}$ anonimato e a privacidade dos participantes foram resguardados no estudo.

Para a análise estatística, foram utilizadas freqüências simples e relativas, adotando-se, para análise da inter-relação entre as variáveis selecionadas, o teste qui-quadrado. As correlações foram feitas com base no teste de Spearman. Os dados foram avaliados pelo programa SPSS 11.0 Statistical Package for the Social Sciences.

\section{RESULTADOS}

A amostra foi composta de 191 alunos do sexo feminino que tinham em média 21,7 anos (DP = 3,5). A média do índice de massa corporal (IMC) dessas estudantes foi $21,3 \mathrm{~kg} / \mathrm{m}^{2}$ $(\mathrm{DP}=2,1)$, demonstrando que a maioria das alunas apresenta um IMC normal, sendo consideradas eutróficas em relação ao seu peso corporal. Quando avaliada a diferença entre o peso informado e o peso desejado dessas alunas foi visto uma média de insatisfação com o peso de $2,2 \mathrm{~kg}$ $(\mathrm{DP}=3,8)$.

De acordo com os questionários auto-aplicáveis, a média de pontuação para o EAT-26 foi 11,2 pontos ( $D P=10,2)$, para o BITE sintomas foi 7,9 pontos $(\mathrm{DP}=5,2)$, para o $\mathrm{BITE}$ 
gravidade, 1,9 pontos ( $D P=2,3)$ e para o BSQ 81, pontos 7 $(\mathrm{DP}=33,5)$.

Em relação à amostra, observou-se que, na escala BITE sintomas, 135 (70,7\%) alunas apresentaram pontuações dentro dos limites normais, 47 (24,6\%) mostraram estar com nível de sintomas médio, indicando a presença de comportamento alimentar de risco, mas que a aplicação da escala de gravidade do BITE evidenciou que o resultado, na maioria dos casos, não é clinicamente significativo para bulimia.

Ao relacionar a escala de gravidade do BITE com a de sintomas de $9(4,7 \%)$ alunas que apresentavam sintomas elevados para comportamento bulímico, 5 (2,6\%) também foram classificadas com gravidade significativa/intensa na escala gravidade do BITE.

Já na escala BITE gravidade, 179 (93,7\%) estavam dentro dos limites normais, 9 (4,7\%) apresentaram valores significantes e $3(1,6 \%)$ mostraram intensa gravidade. Na escala EAT-26, 164 (85,9\%) das alunas apresentaram um valor até 20 pontos e $27(14,1 \%)$ atingiram pontuações de 21 pontos ou mais.

A Tabela 1 mostra que a imagem corporal (BSQ) não está relacionada à idade - ou, indiretamente, à evolução no curso - nem ao IMC. Por outro lado, observa-se uma associação estatisticamente significativa do BSQ com a insatisfação com o peso, o que é demonstrado pelo fato de a prevalência de BSQ moderado/grave atingir quase 30\% entre aquelas que querem perder mais de $2 \mathrm{~kg}$.

Tabela 1. BSQ segundo algumas características das estudantes de educação física

\begin{tabular}{|c|c|c|c|c|c|c|c|}
\hline \multirow{3}{*}{ Caracteristicas } & \multirow{2}{*}{\multicolumn{2}{|c|}{ Total }} & \multicolumn{4}{|c|}{ BSQ } & \multirow{3}{*}{$p$-valor } \\
\hline & & & \multicolumn{2}{|c|}{ Normal/leve } & \multicolumn{2}{|c|}{ Moderado/grave } & \\
\hline & $\mathrm{N}$ & $\%$ & $\mathrm{~N}$ & $\%$ & $\mathrm{~N}$ & $\%$ & \\
\hline \multicolumn{8}{|l|}{ Idade } \\
\hline 17 a 19 anos & 40 & 100,0 & 33 & 82,5 & 7 & 17,5 & \multirow{4}{*}{0,134} \\
\hline 20 ou 21 anos & 79 & 100,0 & 61 & 77,2 & 18 & 22,8 & \\
\hline 22 anos ou mais & 68 & 100,0 & 61 & 89,7 & 7 & 10,3 & \\
\hline Total & 187 & 100,0 & 155 & 82,9 & 32 & 17,1 & \\
\hline \multicolumn{8}{|l|}{$\operatorname{IMC}\left(\mathrm{kg} / \mathrm{m}^{2}\right)$} \\
\hline Até 17,99 & 5 & 100,0 & 5 & 100,0 & 0 & 0,0 & \multirow{4}{*}{0,399} \\
\hline 18 a 24,99 & 171 & 100,0 & 142 & 83,0 & 29 & 17,0 & \\
\hline 25 ou mais & 11 & 100,0 & 8 & 72,7 & 3 & 27,3 & \\
\hline Total & 187 & 100,0 & 155 & 82,9 & 32 & 17,1 & \\
\hline \multicolumn{8}{|l|}{ Insatisfaçäo com o peso** } \\
\hline Querem ganhar peso & 32 & 100,0 & 32 & 100,0 & 0 & 0,0 & \multirow{5}{*}{$<0,001$} \\
\hline Satisfeitas & 25 & 100,0 & 24 & 96,0 & 1 & 4,0 & \\
\hline Querem perder até $2 \mathrm{~kg}$ & 22 & 100,0 & 21 & 95,5 & 1 & 4,5 & \\
\hline $\begin{array}{l}\text { Querem perder mais } \\
\text { de } 2 \mathrm{~kg}\end{array}$ & 105 & 100,0 & 75 & 71,4 & 30 & 28,6 & \\
\hline Total & 184 & 100,0 & 152 & 82,6 & 32 & 17,4 & \\
\hline
\end{tabular}

${ }^{*}$ Teste $\boldsymbol{\chi}_{;}^{2}$ ** peso informado menos peso ideal.
Percebe-se, na Tabela 2, forte associação - estatisticamente significativa - entre comportamento alimentar (EAT-26) e imagem corporal (BSQ). A prevalência de escores elevados no EAT-26 é cerca de 20 vezes maior entre aquelas com BSQ moderado/grave em relação àquelas com BSQ normal/leve (65,6\% para 3,8\%). A Tabela 3 permite conclusão análoga à apontada pela da Tabela 1, evidenciando que o comportamento alimentar (tal como a imagem corporal) mostra-se associado estatisticamente apenas à insatisfação com o peso.

Tabela 2. EAT-26 e BSQ das estudantes de educação física

\begin{tabular}{|c|c|c|c|c|c|c|c|}
\hline \multirow{3}{*}{ EAT-26 } & \multirow{2}{*}{\multicolumn{2}{|c|}{ Total }} & \multicolumn{4}{|c|}{ BSQ } & \multirow{3}{*}{ p-valor* } \\
\hline & & & \multicolumn{2}{|c|}{ Normal/leve } & \multicolumn{2}{|c|}{ Moderado/grave } & \\
\hline & N & $\%$ & N & $\%$ & N & $\%$ & \\
\hline Até 20 pontos & 164 & 85,9 & 153 & 96,2 & 11 & 34,4 & \multirow{2}{*}{$<0,001$} \\
\hline 21 ou mais & 27 & 14,1 & 6 & 3,8 & 21 & 65,6 & \\
\hline Total & 191 & 100,0 & 159 & 100,0 & 32 & 100,0 & \\
\hline
\end{tabular}

Tabela 3. EAT-26 segundo algumas características das estudantes de educação física

\begin{tabular}{|c|c|c|c|c|c|c|c|}
\hline \multirow{3}{*}{ Características } & \multirow{2}{*}{\multicolumn{2}{|c|}{ Total }} & \multicolumn{4}{|c|}{ ЕАТ-26 } & \multirow{3}{*}{ p-valor } \\
\hline & & & \multicolumn{2}{|c|}{ Até 20 pontos } & \multicolumn{2}{|c|}{21 ou mais } & \\
\hline & N & $\%$ & N & $\%$ & N & $\%$ & \\
\hline \multicolumn{8}{|l|}{ Idade } \\
\hline 17 a 19 anos & 40 & 100,0 & 35 & 87,5 & 5 & 12,5 & \multirow{4}{*}{0,303} \\
\hline 20 ou 21 anos & 79 & 100,0 & 64 & 81,0 & 15 & 19,0 & \\
\hline 22 anos ou mais & 68 & 100,0 & 61 & 89,7 & 7 & 10,3 & \\
\hline Total & 187 & 100,0 & 160 & 85,6 & 27 & 14,4 & \\
\hline \multicolumn{8}{|l|}{$\operatorname{IMC}\left(\mathbf{k g} / \mathbf{m}^{2}\right)$} \\
\hline Até 17,99 & 5 & 100,0 & 5 & 100,0 & 0 & 0,0 & \multirow{4}{*}{0,615} \\
\hline 18 а 24,99 & 171 & 100,0 & 146 & 85,4 & 25 & 14,6 & \\
\hline 25 ou mais & 11 & 100,0 & 9 & 81,8 & 2 & 18,2 & \\
\hline Total & 187 & 100,0 & 160 & 85,6 & 27 & 14,4 & \\
\hline \multicolumn{8}{|l|}{ Insatisfação com o peso** } \\
\hline Querem ganhar peso & 32 & 100,0 & 30 & 93,8 & 2 & 6,3 & \multirow{4}{*}{0,005} \\
\hline Satisfeitas & 25 & 100,0 & 25 & 100,0 & 0 & 0,0 & \\
\hline Querem perder até $2 \mathrm{~kg}$ & 22 & 100,0 & 21 & 95,5 & 1 & 4,5 & \\
\hline $\begin{array}{l}\text { Querem perder mais } \\
\text { de } 2 \mathrm{~kg}\end{array}$ & 105 & 100,0 & 82 & 78,1 & 23 & 21,9 & \\
\hline Total & 184 & 100,0 & 158 & 85,9 & 26 & 14,1 & \\
\hline
\end{tabular}

*Teste $\chi^{2 ;}$; ${ }^{* *}$ peso menos peso ideal.

\section{DISCUSSÃO}

Os resultados obtidos revelam expressiva freqüência de comportamentos alimentares considerados anormais no grupo em análise. Segundo Oliveira27, "esses hábitos podem representar o desenvolvimento de síndromes parciais 
de TCA, sendo caracterizados como comportamentos alimentares e de risco francamente anormais".

A literatura aponta ser comum, entre mulheres, a adoção de práticas alimentares e de redução de peso corporal inadequadas, quando insatisfeitas com sua imagem corporal. Essa insatisfação é manifesta em seu desejo de perder peso ${ }^{26,27}$. Não obstante, a distorção da imagem corporal constitui importante sintoma dos TCA, tendo influência da experiência do indivíduo com seu peso e de sua forma corporal28.

É também motivo de preocupação a pontuação obtida no BITE pelas estudantes, que indica expressiva presença de comportamentos alimentares de risco, pois, embora não apontando gravidade em sua maioria, não se descarta, naqueles casos em que intensidade e gravidade são moderadas, a possibilidade de evolução para comportamentos de alta gravidade e para instalação de TCA 29,30.

Em estudo realizado com estudantes de educação física do sexo feminino na Nova Zelândia, O'Brien e Hunter ${ }^{31}$ encontraram, maior prevalência de transtornos alimentares e auto-estima reduzida, quando comparadas com estudantes de outros cursos, expressas pela insatisfação com sua atratividade sexual, com sua condição física e com seu peso.

No estudo de Bosi et al. ${ }^{32}$, realizado com estudantes de nutrição, o escore médio do BSQ foi de 81,2 pontos ( $\pm 33,6)$, correspondendo à preocupação leve com a auto-imagem corporal. A prevalência dessas universitárias com distorção grave da imagem corporal foi de 6,2\% (IC 95\%; 3,2\%-10,6\%) e, segundo os autores, a prevalência de TCA em mulheres varia em função de suas atividades profissionais ou cotidianas.

Nesses termos, os profissionais das áreas correlatas à saúde e/ou à estética - os educadores físicos tangenciam os dois campos - integram um grupo altamente vulnerável ao desenvolvimento de TCA.

\section{CONCLUSÃO}

O estudo em questão aponta que uma parcela significativa das universitárias estudadas apresenta comportamento alimentar fora do normal e deseja perder peso. Tal achado parece de grande relevância, uma vez que a análise do IMC associado ao comportamento alimentar mostra que esse desejo não corresponde ao corpo em suas dimensões objetivas, uma vez que as informantes situam-se rigorosamente na faixa de peso adequada para sua estatura. Tais resultados sinalizam a necessidade de um olhar mais atento, pois sugerem a preponderância dos valores relacionados à estética em detrimento daqueles relacionados à saúde.

\section{AGRADECIMENTOS}

Esta pesquisa contou com apoio financeiro do CNPq, Edital Universal 2003 e da Fundação José Bonifácio/UFRJ.

\section{REFERÊNCIAS}

1. Fairburn CG, Harrison PJ. Eating disorders. Lancet. 2003;361:407-16.

2. Ung EK Eating disorders in Singapore: coming of age. Singapore Med Journal. 2005;46(6):255.

3. Bosi MLM, Palha F0. Comportamentos bulímicos em atletas adolescentes corredoras de fundo. Rev Bras Psiquiatr. 2004;26(1):32-4.

4. Walsh JME, Wheat ME, Freund K. Detection, evaluation and treatment of eating disorders. J Gen Intern Med. 2000;15:577-90.

5. Klein DA, Walsh BT. Eating disorders: clinical features and pathophysiology. Physiology \& Behavior. 2004;81:359-74.

6. Mellin P, Araújo AM. Transtornos alimentares em homens: um desafio diagnóstico. Rev Bras Psiquiatr. 2002;24(Supl III):73-6.

7. Pinzon V, Nogueira FC. Epidemiologia, curso e evolução dos transtornos alimentares. Rev Psiq Clin. 2004;31(4):158-60.

8. American Psychiatric Association (APA). Diagnostic and statistical manual of mental disorder. $4^{a}$ ed. Washington DC: Am Psychiatric Press, 1994.

9. Beatty D, Finn SC. Position of the American Dietetic Association and the Canadian Dietetic Association: women's health and nutrition. J Am Diet Assoc. 1995;95:362-6.

10. Cordás TA, Castilho S. Imagem corporal nos transtornos alimentares. Instrumento de avaliação: Body Shape Questionnaire. Psiquiatr Biol. 1994;2:17-21.

11. Fiates GMR, Salles RK. Fatores de risco para o desenvolvimento de distúrbios alimentares: um estudo em universitárias. Rev Nutr. 2001;14 Suppl:S3-6.

12. Moya T, Fleitlich-Bilyk B, Goodman R, Nogueira FC, Focchi PS, Nicoletti M, et al. The Eating Disorders Section of the Development and Well-Being Assessment (DAWBA): development and validation. Rev Bras Psiquiatr. 2005;27(1):25-31.

13. Sudi K, Öttl K, Payerl D, Baumgartl P, Tauschmann K, Müller W. Anorexia athletica. Nutrition. 2004;20:657-61.

14. Pardini DP. Alterações hormonais da mulher atleta. Arq Bras Endocrinol Metab. 2001;45(4):343-51.

15. Torstveit MK, Sundgot-Borgen J. The female athlete triad: are elite athletes at increased risk? Medicine \& Science in Sports \& Exercise. 2005;37(2):184-93.

16. Cordás TA, Neves JEP. Escalas de avaliação de transtornos alimentares. Rev Psiq Clin. 1999;26:41-8.

17. Garner D, Garfinkel P. Eating attitudes test: an index of the symptoms of anorexia nervous. Psychol Med. 1979;9:273-9.

18. Mann AH, Wakeling A, Wood K, Monck E, Dobbs R, Szmuller G. Screening of abnormal eating attitudes and psychiatric morbidity in unselected population of 15 -year old schoolgirls. Psychol Med. 1983;13:573-80.

19. Johnson-Sabine E, Wood K, Patton G, Mann A, Wakeling A. Abnormal eating attitudes in London schoolgirls. A prospective epidemiological study: factors associated with abnormal response on screening questionnaires. Psychol Med. 1988;18:615-22.

20. Nunes MAA. Prevalência de comportamentos alimentares anormais e práticas inadequadas de controle de peso em mulheres de 12 a 21 anos em Porto Alegre [dissertação]. Porto Alegre, RS: Universidade Federal de Pelotas, 1997.

21. Assunção SSM, Cordás TA, Araújo LASB. Atividade física e transtornos alimentares. Rev Psiq Clin. 2002:29:4-13.

22. Hendrson M, Freeman CPL. A self-rating scale for bulimia - the BITE. Br J Psychiatry. 1987;50:18-24. 
23. Cordás TA, Hpchgraf PB. 0 "BITE": instrumento para avaliação da bulimia nervosa versão para o português. J Bras Psiquiatr. 1993;42:141-4.

24. Cooper P, Taylor MJ, Cooper Z, Fairburn CG. The development and validation of the Body Shape Questionnaire. International Journal of Eating Disorders. 1987;6:485-94.

25. Oliveira FP, Bosi MLM, Vigario PS, et al. Comportamento alimentar e imagem corporal em atletas. Rev Bras Med Esporte. 2003;9(6):348-56.

26. Souto S, Ferro-Bucher, JSN. Práticas indiscriminadas de dietas de emagrecimento e o desenvolvimento de transtornos alimentares. Rev Nutr. 2006;19(6):693-704.

27. Strickland A. Body image and self-esteem: a study of relationships and comparisons between more and less physically active college women. Tallahassee (FL): Florida State Univ., 2004.
28. Saikali CJ, Soubhia CS, Scalfaro BM, Cordás TA. Imagem corporal nos transtornos Alimentares. Rev Psiq Clin. 2004;31(4):164-6.

29. King MA. Eating disorders in a general practice population: prevalence, characteristics and follow-up at 12 to 18 months. Psychol Med. 1989;14 Suppl;S211-8.

30. Patton GC. The spectrum of eating disorders in adolescence. J Psychosom Res. 1988; 32:579-84.

31. O'Brien KS, Hunter JA. Body esteem and eating behaviours in female physical education students. Eat Weight Disord. 2006 Jun;11(2):e57-60.

32. Bosi MLM, Raggio LR, Morgado CMC, Costa MLSS, Carvalho RJ. Autopercepção da imagem corporal entre estudantes de nutrição: um estudo no município do Rio de Janeiro. J Bras Psiquiatr. 2006;55(2): 108-113. 